\title{
On the line shift and oscillator strength of Xe II lines in the spectra of HnMn stars
}

\author{
H. O. Di Rocco ${ }^{1,2}$, A. Cruzado ${ }^{3,4}$, and P. E. Marchiano ${ }^{3}$ \\ 1 Intituto de Física Arroyo Seco (IFAS), Centro de Investigaciones en Física e Ingeniería del Centro de la Provincia de Buenos Aires \\ (CIFICEN), Universidad Nacional del Centro de la Provincia de Buenos Aires (UNCPBA)-Consejo Nacional de Investigaciones \\ Científicas y Técnicas (CONICET), Pinto 399, 7000 Tandil, Buenos Aires, Argentina \\ e-mail: hdirocco@exa.unicen.edu.ar \\ 2 Consejo Nacional de Investigaciones Científicas y Técnicas (CONICET) 1917, Argentina \\ ${ }^{3}$ Facultad de Ciencias Astronómicas y Geofísicas, Univesidad Nacional de La Plata, Paseo del Bosque s/n, 1900 La Plata, \\ Buenos Aires, Argentina \\ e-mail: acruzado@fcaglp.unlp.edu.ar \\ ${ }^{4}$ Instituto de Astrofísica de La Plata (CONICET), Paseo del Bosque s/n, 1900 La Plata, Buenos Aires, Argentina
}

Received 12 April 2015 / Accepted 3 July 2015

\begin{abstract}
Aims. The ultimate goal that has motivated this work is to achieve realistic oscillator strength $(g f)$ values to find reliable values of stellar abundances. We aim to compare the $g f$ valúes of Xe II lines found with different theoretical and experimental methods. Methods. We have undertaken calculations using the quasirelativistic and relativistic Hartree-Fock methods. Then we compare these results with those previously obtained from UVES spectra of HgMn stars.

Results. 1) Our theoretical $g f$ values are more realistic than those previously obtained for most lines. When we consider only unblended, isolated, relatively noise-free lines, however, our theoretical $g f$ values and Yuce's stellar values differ little from each other. 2) In a discussion of the origin of the previously observed discrepancy between the wavelengths of Xe II lines deducted from stellar spectra and those published by National Institute of Standards and Technology (NIST), we conclude that stellar wavelengths could be considered the standard wavelengths whenever the densities in stellar atmospheres are smaller than $1 \times 10^{16}$ part. $\mathrm{cm}^{-3}$.
\end{abstract}

Key words. atomic data - line: identification - stars: abundances - stars: chemically peculiar

\section{Introduction}

Not long ago, Yuce et al. (2011) published a work where oscillator strengths of $100 \mathrm{Xe}$ II lines were inferred from UVES spectra of four HgMn stars: HR 6000, HD 71066, 46 Aql, and HD 175640. With the starting model parameters derived from photometry, the authors used, in turn, the codes ATLAS9, SYNTHE, ATLAS12, and WIDTH to obtain synthetic spectra and abundances of many elements and ions, among them Xe II. In principle, this work is the more extensive source of "experimental" oscillator strength $(g f)$ values for Xe II, as can be verified by comparing with the National Institute of Standards and Technology (NIST) database.

In addition, Yuce et al. (2011) considered blueshifts up to $0.1 \AA$ from the predicted wavelength of some Xe II lines (according to the established level scheme), of a priori unidentified stellar lines. Since similar shifts are characteristic of the high current discharges used in many laboratories to obtain Xe spectra, the data of Yuce et al. (2011) provide a good standard to compare laboratory measurements affected by plasma effects (see below).

With respect to the works made about the Xe II spectrum, since the early papers published in the ' 30 s by Humphreys et al. (1931), Boyce (1936), and Humphreys (1939), several works have been completed regarding energy levels and spectrum of Xe II, transitions probabilities, line shifts, and widths. On the basis of the published material, Hansen \& Persson (1987) reported a revised and complete analysis of the data. Extensive analysis of Xe spectra were performed at the Centro de Investigaciones Ópticas (CIOP, La Plata, Argentina). Some of these works (Persson et al. 1988; Gallardo et al. 1993) are related to Xe II, and they were used as sources of data by Saloman (2004) in his critical compilation.

In particular for Xe II, several works have been published by different authors from 1986 to date. A complete database can be found at the NIST, compiled by Kramida \& Fuhr (2015).

It is important to highlight that most of the Xe II lines analyzed in the article of Yuce et al. (2011) are weak, noised and/or blended, as it is viewed on the web page of Castelli (2011), where the UVES spectra of the stars are shown. In the last section, we analyze the behavior of some lines that are referred by the authors as without blend or noise in the stellar spectra of HR 6000 (see Table 2).

We are very interested in achieving realistic $g f$ values to find reliable values of stellar abundances, since inaccuracies in the values of $g f$ directly translate into inaccuracies in the calculation of stellar abundances. Therefore, we focus on analyzing and comparing the $g f$ values of Xe II lines obtained with different methods, both theoretical and experimental. Since the $g f$ values of Yuce et al. (2011) disagree, in general, with other experimental and theoretical works (Di Rocco et al. 2000, and references therein), we have undertaken new calculations using the quasirelativistic Hartree-Fock and relativistic Hartree-Fock 
methods, which are mandatory approaches because of the relatively high $\mathrm{Z}$ of $\mathrm{Xe}$.

We organize this paper as follows: in Sect. 2 we describe the technique we have used in obtaining the atomic Xe II structure and spectra; in Sect. 3 we present our work methodology and our results; and in Sect. 4 we present our conclusions.

\section{Obtaining the structure and spectra of Xe II}

As is well known in modern spectroscopic analysis, once a number of levels have been found by empirical spectrum analysis, the theory can be used to calculate "the best" possible wavefunctions and, from these, radiative and collisional parameters ( $g f$ values, excitation, and ionization cross sections, etc.). Of paramount importance in the theoretical calculations is the concept of configuration interaction. The "experimental" wavefunctions are expressed in terms of the Hartree-Fock orbitals, which are taken as the base functions. So, the configurations $5 \mathrm{~s} 5 \mathrm{p} 6$ and $5 \mathrm{~s} 25 \mathrm{p} 4(6 \mathrm{~s}+7 \mathrm{~s}+8 \mathrm{~s}+5 \mathrm{~d}+6 \mathrm{~d}+7 \mathrm{~d}+\ldots)$, for example, can be treated together and pure designations are, many times, of little or null significance. Then, a given $|\gamma J\rangle$ level is expressed as $|\gamma J\rangle=\sum_{\beta} y_{\beta j}^{\gamma}|\beta J\rangle$ in terms of the H-F base functions $|\beta J\rangle$ (Cowan 1981). It is expected that an expansion with a manageable small set of $|\beta J\rangle$ functions can be sufficient to give reasonable $g f$ values if the configurations are judiciously chosen (especially if the configurations have the same principal quantum number and/or the energies of the configurations are similar).

Taking into account that the empirical structure of Xe II is well known (Hansen \& Persson 1987), we have proceeded to perform calculations using the set of versatile codes from Cowan (1981). These codes are based on a quasirelativistic configuration interaction approach, allowing a least-squares fitting of the levels. With these codes, we worked in three different ways: i) We consider the electrostatic Slater parameters, $F^{2}(5 \mathrm{p} ; 5 \mathrm{p})$, $F^{2}(5 \mathrm{p} ; \mathrm{nl}), G^{k}(5 \mathrm{p} ; n l)$, and $R^{k}(i j ; t u)$, and the spin-orbit parameter, $\phi_{n l}$, from the least-squares adjustment of the levels (semiempirical analysis) of Hansen \& Persson (1987); we call this the Hansen-Persson least-squares (HPLS) approach. ii) We select the same configurations of Hansen \& Persson (1987), but perform the purely ab initio calculations with proper scaling factors; we call this the Hansen-Persson Ab Initio (HPAI) approach. iii) We use an extended set of congurations judiciously chosen in a purely theoretical form; we call this the Ab Initio Many Congurations (AIMC) approach.

The theoretical results, obtained through the HPLS, HPAI, and AIMC approaches, have already been analyzed and compared, among themselves, and with the experimental approaches; e.g., Di Rocco et al. (2000). Reaffirming their conclusions, we find that the three approaches are all reasonably consistent and, therefore, some degree of quality can be conferred to all our calculations. Given this, we may choose any set of theoretical results to compare them with the results of Yuce et al. (2011), which is our aim. We may also take into consideration all of our theoretical results and obtain a final result as

$g f=\tilde{g f} \pm \frac{\sqrt{\sum_{i}\left(\tilde{g f}-(g f)_{i}\right)^{2}}}{N}$.

Furthermore, we have used the code FAC from Gu (2008). This is a fully relativistic MultiConfiguration Dirac-Hartree-Fock (MCDHF) approach, widely used in recent years. It is important to highlight that the two sets of $g f$ values, obtained with
FAC and Cowan codes, are very similar. Clearly, we can use the $g f$ values of different codes and generalize the Eq. (1).

With that in mind, the aim of this article can be achieved by comparing the theoretical results obtained with either approach outlined above with those of Yuce et al. (2011). Therefore, to simplify and streamline our research, we use the results we obtained with the HPLS approach to work.

\section{Calculations and results}

\subsection{The calculation of the oscillator strengths}

Following Cowan's nomenclature (1981), the calculation of transition probabilities, $A_{i j}$, and related weighted oscillator strengths, $g f_{i j}$, are related to the calculation of line strength

$\boldsymbol{S}_{i j}=\left|\left\langle\gamma J\left\|\boldsymbol{P}^{(1)}\right\| \gamma^{\prime} J^{\prime}\right\rangle\right|^{2}$

where $\boldsymbol{P}^{(1)}$ is the dipole moment of the atom measured in units of $-e a_{0}$. When the energies are measured in Rydbergs, the relation between $g f$ and $S$ is given by

$g f=\frac{E_{j}-E_{i}}{3} S$

The states $|\gamma J\rangle=\sum_{i} a_{i} \phi_{i}$ and $\left|\gamma^{\prime} J^{\prime}\right\rangle=\sum_{i} a_{i}^{\prime} \phi_{i}^{\prime}$ can be constructed basically in two ways: configuration interaction and multiconfiguration expansions. In the first case, the coefficients $a_{i}$ are fixed by the theory and the $\phi_{i}$ are optimized; in the second case, both the $a_{i}$ and the $\phi_{i}$ are optimized. The more useful codes using the CI approach are Cowan's (quasirelativistic) and Gu's (relativistic) codes; among others, the MCDHF approach is due to Froese Fischer (1997) and Grant (2007). We use the two CI treatments from Cowan and $\mathrm{Gu}$ because both of those are free and straightforward to use. In our opinion, the methodology of Cowan is convenient: we can use a least-squares fit of the levels and scale the radial factors (Slater integrals $F^{k}, G^{k}$ and $\zeta_{n l}$ ) in that process. Then, that scaling process indirectly takes several difficult aspects of the many atom theory (exchange, correlation, etc.) into account.

\subsection{A brief about the line widths and shifts}

When atoms and ions are immersed in plasma characterized by their electron temperature, $T_{\mathrm{e}}$, and electron density, $N_{\mathrm{e}}$, the energy levels are shifted with respect to the ideal situation of the "isolated" atom (Griem 1964). Furthermore, the spectral lines are broadened because the collisions with free electrons shorten the lifetime of the excited electrons in the atoms. There are diverse mechanisms of plasma broadening but, because in the laboratory experiments the spectra of Xe II are obtained using high current pinched discharges $\left(T_{\mathrm{e}} \approx 1-2 \mathrm{eV}, N_{\mathrm{e}} \approx 1 \times 10^{16}-1 \times\right.$ $10^{17} \mathrm{~cm}^{-3}$ ), the more important mechanisms are those generically called pressure broadening (in particular, interactions with charged particles), which are treated mathematically via the impact approximation (Griem 1997; Sobelman et al. 1995).

Although we do not make explicit calculations about shifts $(d)$ and widths $(w)$, we recall that the calculation of both, $d$ and $w$, as well as the behavior of $T_{\mathrm{e}}$ and $N_{\mathrm{e}}$, are very complex (see Sahal-Bréchot et al. 2014, as well as Peláez et al. 2009a,b). Summing up, in the semiclassical approximation both, shifts and broadenings, are basically proportional to $N_{\mathrm{e}} T_{\mathrm{e}}^{-1 / 2}$. 
Regarding $d$, whereas for $6 p-6 d$ and $6 p-7 s$ transitions $d \approx$ $0.1 \AA$, for $6 s-6 p$ and $5 d-6 p$ transitions $d$ is, in general, much lower because the initial and final levels are shifted almost the same amount. Stark parameters of some XeII lines were measured by Peláez et al. (2009a,b) for the $6 p-6 d, 6 p-7 s, 6 s-6 p$ and $5 d-6 p$ transitions. For the two last cases, experimental errors are, sometimes, a significant percentage of the measured Stark shifts. For comparison, it is important to establish that in the works of classical atomic spectroscopy, where the lines are measured on photographic plates, the accuracy is, typically, of the order of $0.01 \AA$.

\subsection{Results}

\subsubsection{General trends}

To begin with, we compare the $g f$ values published by Yuce et al. (2011) with our theoretical $g f$ values. From our calculations carried out, including the $5 \mathrm{~s} 25 \mathrm{p} 46 \mathrm{p}, 5 \mathrm{~s} 5 \mathrm{p} 6,5 \mathrm{~s} 25 \mathrm{p} 46 \mathrm{~s}$, $5 \mathrm{~s} 25 \mathrm{p} 47 \mathrm{~s}, 5 \mathrm{~s} 25 \mathrm{p} 4$ 8s, 5s 2 5p 4 5d, 5s 2 5p 4 6d, and 5s2 5p4 7d configurations, we have been able to identify 91 of the 98 lines for which Yuce et al. (2011) have found stellar $g f$ values. In Table 1, third and fourth columns, the stellar $g f$ values, obtained by Yuce et al. (2011), and the theoretical values we obtained with the Cowan Code, are shown for these 91 lines. For these 91 Xe II lines, we analyze, at first, the ratio between our theoretical $g f$ values and the $g f$ values inferred by Yuce et al. (2011) from UVES spectra of HgMn stars, $g f_{\text {th }} / g f_{\text {st. }}$. From this preliminary analysis, it has been apparent that some lines are too far away from the theoretical values to be adjusted. Therefore, we eliminate seven lines for which the ratio $g f_{\text {th }} / g f_{\text {st }}>6$ from any subsequent analysis. Our intention is not to do a line-by-line comparison, but rather to assess general trends and behaviors.

We believe that, perhaps, the shift between the stellar and theoretical $g f$ values for the remaining 84 lines could be reduced by adjusting the radial integrals entering the $g f$ calculation. We adjusted these radial integrals for each pair of atomic configurations to achieve the best possible fit for all lines arising from the pair. In Fig. 1, panels a) and b), we show two possible fits. In both panels, the logarithm of stellar $g f$ values are shown as a function of the theoretical values. The adjustment for the 84 Xe II lines is shown in panel a). In an attempt to improve this adjustment, we eliminted nine lines and obtained the fit shown in panel b). In Table 1, fifth and sixth columns, the theoretical $g f$ values are displayed for the 84 and 75 lines used in the first and second adjustment, respectively. Two important things have to be pointed out. First, adjusting the radial integrals does not significantly improve the correlation. In any case, the linear correlation coefficients take values between $r=0.61$ and $r=0.63$, as well as when nine lines are eliminated. Second, the factors that multiply the radial integrals range from 1.15 to 1.9 vary very little from one adjustment to another. Even if the correlation had improved, these factors that multiply the integrals are too large to account for the differences between the theoretical $g f$ values to the stellar values.

In order to find some general trends in the ratio $g f_{\mathrm{th}} / g f_{\mathrm{st}}$, in Fig. 2 this ratio is shown as a function of $\lambda$. In panel a) the 84 lines are included. In panel b) seven lines have been removed to demonstrate that a clear correlation exists between the ratio $g f_{\text {th }} / g f_{\text {st }}$ and $\lambda$. From Fig. 2 it seems that some systematic source of error is affecting the results.
Table 1. Stellar and theoretical $g f$ values.

\begin{tabular}{|c|c|c|c|c|c|}
\hline $\begin{array}{l}\lambda \\
(\AA)\end{array}$ & Conf. & $\log \left(g f_{\mathrm{st}}\right)$ & $\log \left(g f_{\mathrm{th}}\right)$ & $\log \left(g f_{\mathrm{th}, \mathrm{ad} 1}\right)$ & $\log \left(g f_{\text {th,ad} 2}\right)$ \\
\hline 3907.820 & $6 \mathrm{p} 6 \mathrm{~d}$ & -0.82 & -0.1220 & -0.6636 & -0.6827 \\
\hline 4037.260 & $6 \mathrm{p} 6 \mathrm{~d}$ & -1.00 & -0.8190 & -1.3597 & -1.3788 \\
\hline 4037.470 & $6 p 6 d$ & -0.75 & -0.0710 & -0.6126 & -0.6317 \\
\hline 4057.360 & $6 \mathrm{p} 6 \mathrm{~d}$ & -0.80 & -0.3240 & -0.8658 & -0.8849 \\
\hline 4157.980 & $6 \mathrm{p} 6 \mathrm{~d}$ & -0.60 & 0.0280 & -0.5122 & -0.5313 \\
\hline 4162.160 & $5 \mathrm{~d} 6 \mathrm{p}$ & -1.57 & -0.9460 & -1.0668 & -1.0992 \\
\hline 4180.007 & $6 \mathrm{~d} 6 \mathrm{p}$ & -0.35 & 0.2350 & -0.3060 & -0.3251 \\
\hline 4193.100 & $6 d 6 p$ & -0.60 & 0.6950 & & \\
\hline 4208.391 & $6 d 6 p$ & -0.38 & 0.1520 & -0.3893 & -0.4084 \\
\hline 4209.370 & $6 \mathrm{~d} 6 \mathrm{p}$ & -0.70 & -0.1420 & -0.6836 & -0.7027 \\
\hline 4213.620 & $6 \mathrm{p} 6 \mathrm{~d}$ & -0.22 & 0.3250 & -0.2173 & -0.2364 \\
\hline 4215.620 & $6 s 6 p$ & -1.05 & -0.6390 & -0.8524 & -0.8944 \\
\hline 4222.900 & $6 \mathrm{p} 6 \mathrm{~d}$ & 0.64 & 0.1220 & -0.4210 & \\
\hline 4238.135 & $6 \mathrm{p} 6 \mathrm{~d}$ & -0.23 & 0.3240 & -0.2173 & -0.2364 \\
\hline 4245.300 & $6 \mathrm{p} 6 \mathrm{~d}$ & -0.13 & 0.5110 & -0.0310 & -0.0501 \\
\hline 4251.540 & $6 \mathrm{p} 6 \mathrm{~d}$ & -0.58 & 0.1150 & -0.4276 & -0.4467 \\
\hline 4330.390 & $6 \mathrm{p} 6 \mathrm{~d}$ & 0.30 & 0.5200 & -0.0217 & -0.0408 \\
\hline 4369.100 & $6 \mathrm{p} 6 \mathrm{~d}$ & -0.72 & -0.1500 & -0.6915 & -0.7106 \\
\hline 4373.700 & $6 \mathrm{p} 6 \mathrm{~d}$ & -0.70 & -0.1370 & -0.6788 & -0.6979 \\
\hline 4393.090 & $6 \mathrm{p} 6 \mathrm{~d}$ & 0.00 & 0.5330 & -0.0088 & -0.0279 \\
\hline 4395.770 & $6 \mathrm{p} 6 \mathrm{~d}$ & 0.00 & 0.8260 & & \\
\hline 4416.090 & $6 \mathrm{p} 6 \mathrm{~d}$ & -0.80 & 0.1330 & & \\
\hline 4448.025 & $6 \mathrm{p} 6 \mathrm{~d}$ & 0.10 & 0.7160 & 0.1745 & 0.1554 \\
\hline 4462.090 & $6 \mathrm{p} 6 \mathrm{~d}$ & 0.33 & 8190 & 0.2773 & 0.2582 \\
\hline 4787.770 & $5 d 6 p$ & -0.82 & -0.6350 & -0.7544 & -0.7868 \\
\hline 4817.980 & $5 \mathrm{~d} 6 \mathrm{p}$ & -1.25 & -0.9000 & -1.0195 & -1.0519 \\
\hline 4823.250 & $6 \mathrm{p} 7 \mathrm{~s}$ & -0.65 & -0.1070 & -0.4832 & -0.4832 \\
\hline 4844.330 & $6 s 6 p$ & 0.61 & 0.5140 & 0.3004 & \\
\hline 4876.500 & $6 s 6 p$ & 0.10 & 0.2660 & 0.0530 & 0.0110 \\
\hline 4883.530 & $6 s 6 p$ & -0.25 & -0.0010 & -0.2150 & -0.2570 \\
\hline 4884.090 & $6 \mathrm{p} 7 \mathrm{~s}$ & -0.80 & -0.3690 & -0.7450 & -0.7450 \\
\hline 4887.300 & $6 s 6 p$ & -0.85 & -0.4380 & -0.6519 & -0.6939 \\
\hline 4890.085 & $6 s 6 p$ & -1.17 & -0.7690 & -0.9837 & -1.0257 \\
\hline 4919.660 & $5 \mathrm{~d} 6 \mathrm{p}$ & -0.85 & -0.5030 & -0.6229 & -0.6553 \\
\hline 4921.480 & $6 s 6 p$ & 0.05 & 0.3020 & 0.0869 & 0.0449 \\
\hline 4972.700 & $6 s 6 p$ & -0.55 & -0.1220 & -0.3362 & -0.3782 \\
\hline 4988.725 & $5 \mathrm{~d} 6 \mathrm{p}$ & -0.85 & -0.4820 & -0.6014 & -0.6338 \\
\hline 5044.920 & $6 s 6 p$ & -0.80 & -0.2500 & -0.4644 & -0.5064 \\
\hline 5080.510 & $6 \mathrm{p} 7 \mathrm{~s}$ & -0.22 & 0.1380 & -0.2397 & -0.2397 \\
\hline 5122.310 & $6 \mathrm{p} 7 \mathrm{~s}$ & -0.37 & -0.3050 & -0.6818 & -0.6818 \\
\hline 5188.080 & $6 \mathrm{p} 7 \mathrm{~s}$ & -1.10 & 0.1710 & & \\
\hline 5260.420 & $5 \mathrm{~d} 6 \mathrm{p}$ & -0.37 & -0.2750 & -0.3948 & -0.4272 \\
\hline 5261.950 & $6 s 6 p$ & 0.25 & 0.1390 & -0.0743 & \\
\hline 5268.250 & $5 \mathrm{~d} 6 \mathrm{p}$ & -0.80 & -1.1640 & -1.2842 & -1.3166 \\
\hline 5292.220 & $6 s 6 p$ & 0.49 & 0.4290 & 0.2156 & \\
\hline 5309.270 & $6 s 6 p$ & -0.95 & -0.5320 & -0.7458 & -0.7878 \\
\hline 5313.760 & $6 \mathrm{p} 7 \mathrm{~s}$ & -0.09 & 0.2650 & -0.1116 & -0.1116 \\
\hline 5339.355 & $6 s 6 p$ & -0.10 & 0.0540 & -0.1611 & -0.2031 \\
\hline 5368.075 & $5 \mathrm{~d} 6 \mathrm{p}$ & -1.05 & -1.9600 & -2.0785 & -2.1109 \\
\hline 5372.405 & $6 s 6 p$ & -0.15 & -0.1420 & -0.3562 & -0.3982 \\
\hline 5419.155 & $6 s 6 p$ & 0.37 & 0.3460 & 0.1322 & \\
\hline 5438.960 & $6 s 6 p$ & -0.44 & -0.1360 & -0.3503 & -0.3922 \\
\hline 5450.450 & $5 \mathrm{~d} 6 \mathrm{p}$ & -0.97 & -4.4930 & -4.6134 & -4.6458 \\
\hline 5460.365 & $5 \mathrm{~d} 6 \mathrm{p}$ & -0.77 & -0.6010 & -0.7202 & -0.7526 \\
\hline 5472.600 & $5 \mathrm{~d} 6 \mathrm{p}$ & -0.55 & -0.3540 & -0.4735 & -0.5059 \\
\hline 5531.050 & $5 d 6 p$ & -0.78 & -0.6610 & -0.7814 & -0.8138 \\
\hline & $5 \mathrm{~d} 6 \mathrm{p}$ & -0.70 & 0.4582 & & \\
\hline 5659.380 & $6 s 6 p$ & -0.65 & -0.3530 & -0.5668 & -0.6088 \\
\hline
\end{tabular}

Notes. The first and second columns list the wavelength and the configurations which originate the lines. In the third column the values of $\log (g f)$ inferred by Yuce et al. (2011) are shown. In the fourth column the values of $\log (g f)$ given by the code Cowan are indicated. The values of $\log (g f)$ given by the code Cowan after adjusting Slater integrals, for two different groups of lines (see text for explanation), are exhibited in the fifth and sixth columns. 

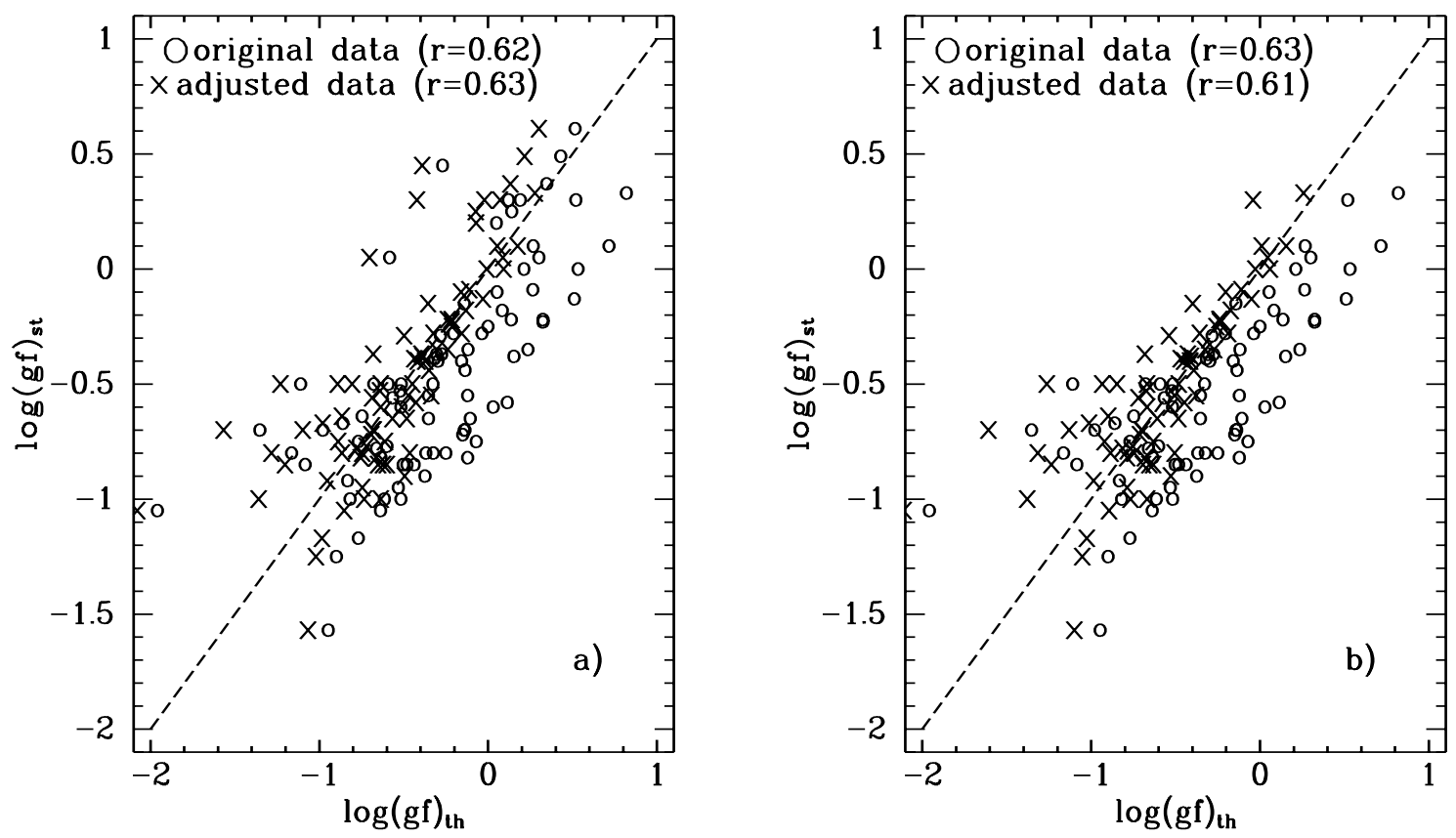

Fig. 1. Logarithm of stellar $g f$ values as a function of the theoretical values. In both panels, the original data before any adjustment are indicated with circles and the data after adjusting the radial integrals are indicated with crosses. In panel a), the adjustment for 84 Xe II lines is shown. In panel b), nine lines have been eliminated (see text).

Table 1. continued.

\begin{tabular}{llcccc}
\hline \hline$\lambda$ & Conf. & $\log \left(g f_{\mathrm{st}}\right)$ & $\log \left(g f_{\mathrm{th}}\right)$ & $\log \left(g f_{\mathrm{th}, \mathrm{ad} 1}\right)$ & $\log \left(g f_{\mathrm{th}, \mathrm{ad} 2}\right)$ \\
$(\AA)$ & & & & & \\
\hline 5667.540 & $5 \mathrm{~d} 6 \mathrm{p}$ & -0.53 & -0.5210 & -0.6413 & -0.6737 \\
5699.610 & $5 \mathrm{~d} 6 \mathrm{p}$ & -0.85 & 0.1811 & & \\
5719.587 & $5 \mathrm{~d} 6 \mathrm{p}$ & -0.80 & 0.2181 & & \\
5726.880 & $5 \mathrm{~d} 6 \mathrm{p}$ & -0.28 & -0.0370 & -0.1570 & -0.1894 \\
5750.990 & $6 \mathrm{~s} 6 \mathrm{p}$ & -0.40 & -0.1580 & -0.3722 & -0.4142 \\
5758.665 & $5 \mathrm{~d} 6 \mathrm{p}$ & -0.35 & -0.1190 & -0.2391 & -0.2715 \\
5776.390 & $5 \mathrm{~d} 6 \mathrm{p}$ & -0.70 & -0.9770 & -1.0987 & -1.1311 \\
5893.290 & $5 \mathrm{~d} 6 \mathrm{p}$ & -0.90 & -0.3750 & -0.4946 & -0.5270 \\
5905.115 & $5 \mathrm{~d} 6 \mathrm{p}$ & -0.75 & -0.7700 & -0.8894 & -0.9218 \\
5945.530 & $5 \mathrm{~d} 6 \mathrm{p}$ & -0.67 & -0.8590 & -0.9800 & -1.0124 \\
5971.135 & $6 \mathrm{~s} 6 \mathrm{p}$ & -0.50 & -0.5920 & -0.8059 & -0.8479 \\
5976.460 & $6 \mathrm{~s} 6 \mathrm{p}$ & -0.29 & -0.2830 & -0.4973 & -0.5393 \\
6036.170 & $5 \mathrm{~d} 6 \mathrm{p}$ & -0.56 & -0.5670 & -0.6869 & -0.7193 \\
6051.120 & $5 \mathrm{~d} 6 \mathrm{p}$ & -0.28 & -0.2050 & -0.3247 & -0.3571 \\
6097.570 & $5 \mathrm{~d} 6 \mathrm{p}$ & -0.39 & -0.3180 & -0.4377 & -0.4701 \\
6101.370 & $5 \mathrm{~d} 6 \mathrm{p}$ & -0.50 & -0.5160 & -0.6356 & -0.6680 \\
6270.810 & $6 \mathrm{~s} 6 \mathrm{p}$ & -0.18 & 0.0810 & -0.1314 & -0.1734 \\
6343.950 & $5 \mathrm{~d} 6 \mathrm{p}$ & -0.64 & -0.7470 & -0.8670 & -0.8994 \\
6375.280 & $5 \mathrm{~d} 6 \mathrm{p}$ & -1.00 & -0.6150 & -0.7343 & -0.7667 \\
6512.790 & $5 \mathrm{~d} 6 \mathrm{p}$ & -1.00 & -0.5170 & -0.6370 & -0.6694 \\
6528.650 & $5 \mathrm{~d} 6 \mathrm{p}$ & -0.40 & -0.2970 & -0.4166 & -0.4490 \\
6594.970 & $5 \mathrm{~d} 6 \mathrm{p}$ & 0.00 & 0.2130 & 0.0923 & 0.0599 \\
6597.230 & $5 \mathrm{~d} 6 \mathrm{p}$ & -0.60 & -0.5170 & -0.6370 & -0.6694 \\
6620.020 & $5 \mathrm{~d} 6 \mathrm{p}$ & -0.85 & -1.0840 & -1.2039 & -1.2363 \\
6694.285 & $5 \mathrm{~d} 6 \mathrm{p}$ & -0.92 & -0.8330 & -0.9526 & -0.9850 \\
6788.710 & $6 \mathrm{~s} 6 \mathrm{p}$ & -0.50 & -0.6770 & -0.8919 & -0.9339 \\
6790.370 & $6 \mathrm{~s} 6 \mathrm{p}$ & -0.70 & -1.3530 & -1.5668 & -1.6088 \\
6990.835 & $5 \mathrm{~d} 6 \mathrm{p}$ & 0.30 & 0.1890 & 0.0705 & \\
7082.150 & $5 \mathrm{~d} 6 \mathrm{p}$ & 0.05 & -0.5830 & -0.7032 & \\
7164.850 & $5 \mathrm{~d} 6 \mathrm{p}$ & 0.20 & 0.0480 & -0.0707 & \\
7284.340 & $5 \mathrm{~d} 6 \mathrm{p}$ & -0.50 & -1.1100 & -1.2300 & -1.2624 \\
7339.300 & $5 \mathrm{~d} 6 \mathrm{p}$ & 0.45 & -0.2700 & -0.3899 & \\
7787.040 & $5 \mathrm{~d} 6 \mathrm{p}$ & -0.50 & -0.3280 & -0.4478 & -0.4802 \\
\hline & & & & & \\
\hline
\end{tabular}

\subsubsection{Individual behavior}

We have taken 32 lines from the set of lines referred by Yuce et al. (2011) as without blend or noise; these are clearly isolated lines in the HR 6000 stellar spectrum (Castelli 2011). Wavelength, $\log \left(g f_{\mathrm{st}}\right), \log \left(g f_{\mathrm{th}}\right)$, as well as $\left|g f_{\mathrm{st}}-g f_{\mathrm{th}}\right| * 100 / g f_{\mathrm{st}}$, are shown in Table 2 for these 32 lines. In Fig. 3 the logarithm of stellar $g f$ values as a function of the theoretical value is shown for these 32 lines. It is clear that the theoretical and stellar values keep a reasonable ratio for these lines $(r=0.87)$. We must not forget that inaccuracies in the values of $g f$ directly translate into inaccuracies in the calculation of stellar abundances.

\section{Conclusions}

The correlation between $g f_{\text {th }} / g f_{\text {st }}$ and $\lambda$, made evident in Fig. 2, can be explained. On the one hand, the weaker a line, the more uncertain its astrophysical $g f$ value. Since the $g f$ values have a tendency to decrease as wavelength increases, the $g f$ values of red lines are, in general, less accurate. On the other hand, the larger the wavelength, the larger the noise and the number of telluric lines in the spectra. This also lowers the accuracy of the results, as pointed out by Yuce et al. (2011). In general, our theoretical results (Table 1, Col. 4) are nearest other authors' theoretical or experimental results than they are to Yuce's results. For that reason, we are able to say that our theoretical $g f$ values are more realistic that those by Yuce el al. (2011). But, when we consider unblended, relatively noise-free, and isolated lines, our theoretical $g f$ values and Yuce's stellar values differ less from each other, as we see in Fig. 3.

Note the systematic shift due to plasma effects (Griem 1964) observed in the wavelength of the lines associated with the $6 \mathrm{~d}$ and $7 \mathrm{~s}$ Xe II energy levels from different spectral sources. Several works have been published on this topic by diverse authors, some as old as that by Di Rocco et al. (1986). Yuce et al. (2011) mention a discrepancy between their wavelengths measured from stellar spectra and those published by NIST, and they 
H. O. Di Rocco et al.: On the line shift and oscillator strength of Xe II lines in stellar spectra
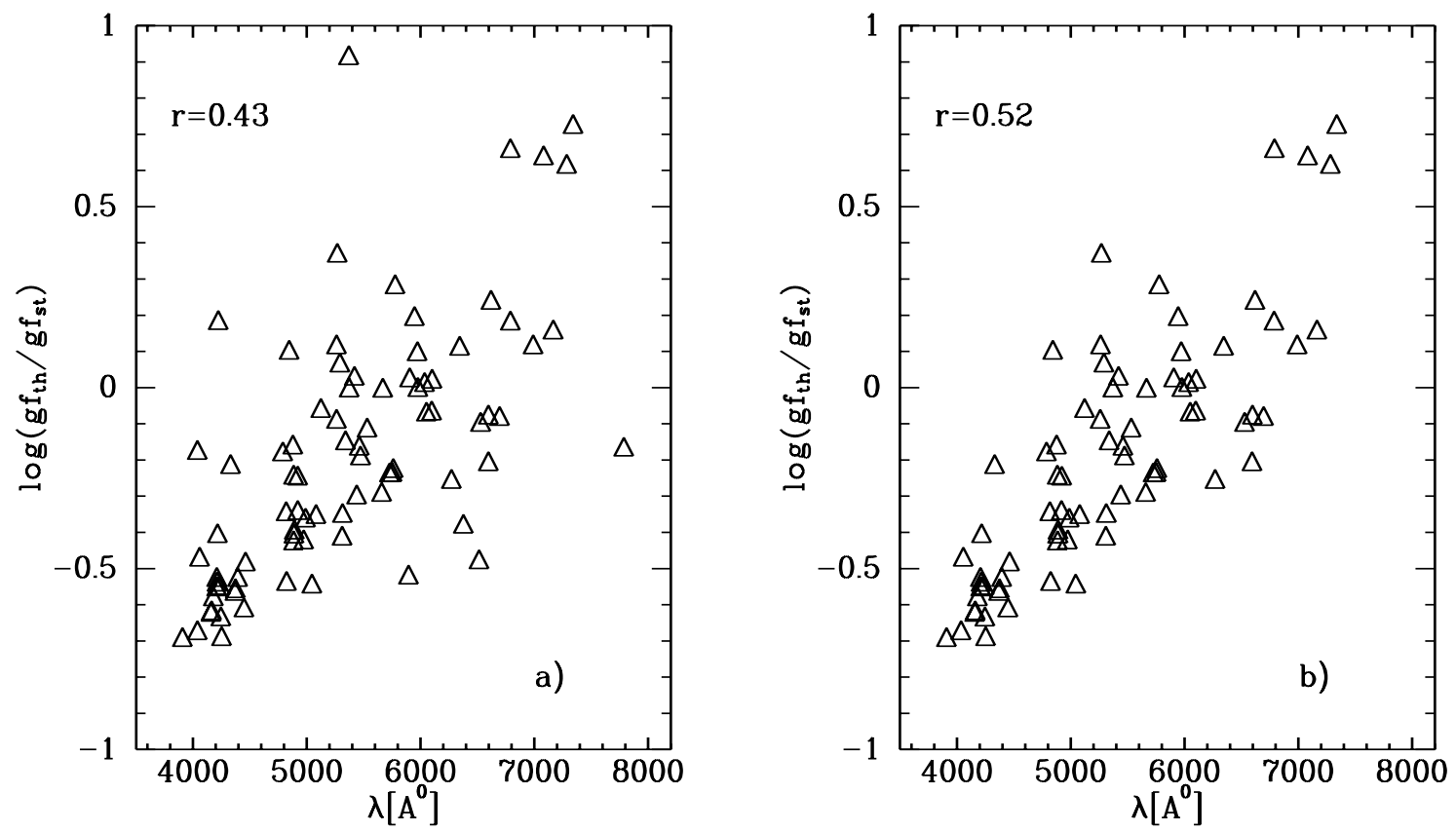

Fig. 2. $g f_{\text {th }} / g f_{\text {st }}$ as a function of $\lambda$. In panel a) 84 lines are included. In panel b) seven lines have been removed (see text).

Table 2. Stellar and theoretical $g f$ values for lines referred by Yuce et al. (2011) as without blend or noise in the HR 6000 stellar spectrum (Castelli 2011).

\begin{tabular}{lcccc}
\hline \hline$\lambda$ & Conf. & $\log \left(g f_{\text {st }}\right)$ & $\log \left(g f_{\text {th }}\right)$ & $\left|g f_{\text {st }}-g f_{\text {th }}\right| / g f_{\text {st }}$ \\
$(\AA)$ & & & & \\
\hline 4208.391 & $5 \mathrm{~d} 6 \mathrm{p}$ & -0.38 & 0.15 & 70.49 \\
4222.900 & $6 \mathrm{p} 6 \mathrm{~d}$ & 0.64 & 0.12 & 231.13 \\
4238.135 & $6 \mathrm{p} 6 \mathrm{~d}$ & -0.23 & 0.32 & 71.82 \\
4330.390 & $6 \mathrm{p} 6 \mathrm{~d}$ & 0.30 & 0.52 & 39.74 \\
4393.090 & $6 \mathrm{p} 6 \mathrm{~d}$ & 0.00 & 0.53 & 70.49 \\
4448.025 & $6 \mathrm{p} 6 \mathrm{~d}$ & 0.15 & 0.72 & 73.08 \\
4817.980 & $5 \mathrm{~d} 6 \mathrm{p}$ & -1.25 & -0.90 & 55.33 \\
4883.530 & $6 \mathrm{~s} 6 \mathrm{p}$ & -0.25 & -0.00 & 43.77 \\
4890.085 & $6 \mathrm{~s} 6 \mathrm{p}$ & -1.17 & -0.77 & 60.19 \\
4919.660 & $5 \mathrm{~d} 6 \mathrm{p}$ & -0.85 & -0.50 & 55.33 \\
4921.480 & $6 \mathrm{~s} 6 \mathrm{p}$ & 0.05 & 0.30 & 43.77 \\
4972.700 & $6 \mathrm{~s} 6 \mathrm{p}$ & -0.55 & -0.12 & 62.85 \\
5080.510 & $6 \mathrm{p} 7 \mathrm{~s}$ & -0.22 & 0.14 & 56.35 \\
5261.950 & $6 \mathrm{~s} 6 \mathrm{p}$ & 0.25 & 0.14 & 28.82 \\
5292.220 & $6 \mathrm{~s} 6 \mathrm{p}$ & 0.49 & 0.43 & 14.82 \\
5313.760 & $6 \mathrm{p} 7 \mathrm{~s}$ & -0.09 & 0.27 & 56.35 \\
5339.355 & $6 \mathrm{~s} 6 \mathrm{p}$ & -0.10 & 0.05 & 29.21 \\
5419.155 & $6 \mathrm{~s} 6 \mathrm{p}$ & 0.37 & 0.35 & 4.71 \\
5438.960 & $6 \mathrm{~s} 6 \mathrm{p}$ & -0.44 & -0.14 & 49.88 \\
5460.365 & $5 \mathrm{~d} 6 \mathrm{p}$ & -0.77 & -0.60 & 32.39 \\
5472.600 & $5 \mathrm{~d} 6 \mathrm{p}$ & -0.55 & -0.35 & 36.90 \\
5531.050 & $5 \mathrm{~d} 6 \mathrm{p}$ & -0.78 & -0.66 & 24.14 \\
5667.540 & $5 \mathrm{~d} 6 \mathrm{p}$ & -0.53 & -0.52 & 2.28 \\
5905.115 & $5 \mathrm{~d} 6 \mathrm{p}$ & -0.75 & -0.77 & 4.71 \\
5976.460 & $6 \mathrm{~s} 6 \mathrm{p}$ & -0.29 & -0.28 & 2.28 \\
6036.170 & $5 \mathrm{~d} 6 \mathrm{p}$ & -0.56 & -0.57 & 2.33 \\
6051.120 & $5 \mathrm{~d} 6 \mathrm{p}$ & -0.28 & -0.20 & 16.82 \\
6097.570 & $5 \mathrm{~d} 6 \mathrm{p}$ & -0.39 & -0.32 & 14.89 \\
6343.950 & $5 \mathrm{~d} 6 \mathrm{p}$ & -0.64 & -0.75 & 28.82 \\
6512.790 & $5 \mathrm{~d} 6 \mathrm{p}$ & -1.00 & -0.52 & 66.89 \\
6694.285 & $5 \mathrm{~d} 6 \mathrm{p}$ & -0.92 & -0.83 & 18.72 \\
6990.835 & $5 \mathrm{~d} 6 \mathrm{p}$ & 0.30 & 0.19 & 28.82 \\
\hline & & & & \\
\hline
\end{tabular}

interpret the origin of this discrepancy as mostly due to incorrect energy levels. We establish now that, indeed, energy levels

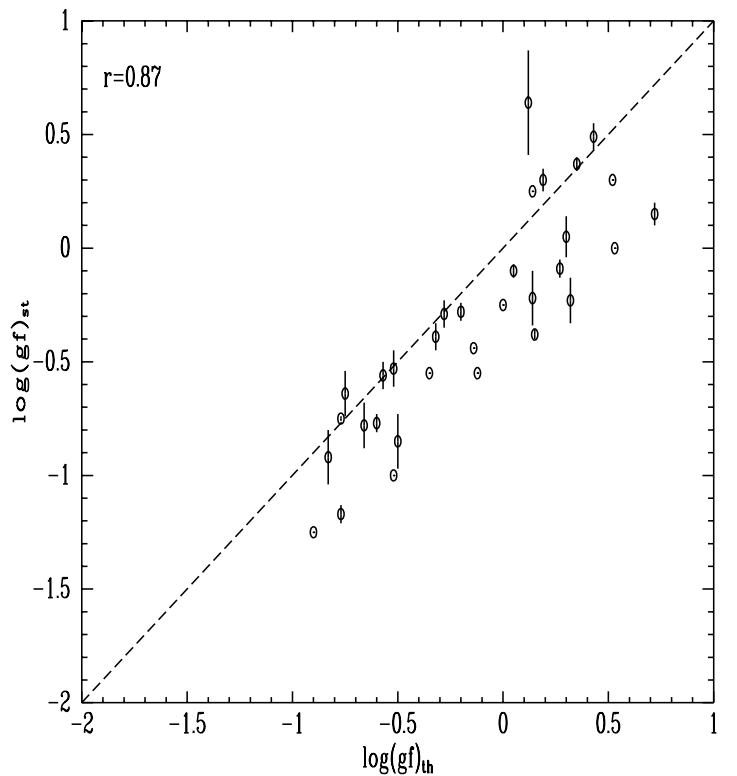

Fig. 3. Logarithm of stellar $g f$ values as a function of the theoretical values for 32 isolated lines (see text). Error bars as given by Yuce et al. (2011) are indicated.

should undergo a shift in laboratory experiments. Therefore, it is clear that these lines are Xe II lines, but shifted by plasma effects, as explained above. In fact, modeling a perturbing potential by an expression of the form $V_{k}(r)=\sum_{k} C_{k} r^{-k}$, we can infer that the energy levels and, therefore, the atomic lines are shifted when the atom is inmersed in a plasma instead of being isolated. The case $k=4$ is of paramount importance in taking collisions with electrons into account. In the semiclassical approximation (Griem 1964), both shifts and broadenings are proportional to $N_{\mathrm{e}} T_{\mathrm{e}}^{-1 / 2}$, where $N_{\mathrm{e}}$ and $T_{\mathrm{e}}$ are the electron density and electron temperature, respectively. In any case, stellar wavelengths could be considered the standard wavelengths whenever the densities in stellar atmospheres are smaller than $1 \times 10^{16}$ part. $\mathrm{cm}^{-3}$. 
Acknowledgements. The authors sincerely appreciate the corrections and suggestions made by the referee whose contributions have greatly improved our article.

\section{References}

Boyce, J. C. 1936 , Phys. Rev., 49, 730

Castelli, F. 2011, http://wwwuser.oats.inaf.it/castelli/stars.html

Cowan, R. D. 1981, The Theory of Atomic Structure and Spectra (Berkeley, Los Angeles, London: University of California Press)

Di Rocco, H. O., Bertuccelli, G., Reyna Almandos, J. G., \& Gallardo, M. 1986, J. Quant. Spectr. Rad. Transf., 35, 443

Di Rocco, H. O., Iriarte, D. I., \& Pomarico, J. A. 2000, EPJD, 10, 19

Froese Fischer, C., Brage, T., \& Jönsson, P. 1997, Computational Atomic Structure (Bristol and Philadelphia: IOP Publishing)

Gallardo, M., Raineri, M., \& Almandos, J. G. Reyna 1993, Spectr. Lett., 26, 1241

Grant, I. P. 2007, Relativistic Quantum Theory of Atoms and Molecules (New York: Springer)

Griem, H. R. 1964, Plasma Spectroscopy (Berkeley, Los Angeles, London: University of California Press)
Griem, H. R. 1997, Principles of Plasma Spectroscopy (Cambridge: Cambridge University Press)

Gu, M. F. 2008, Can. J. Phys., 86, 675

Hansen, J. E., \& Persson, W. 1987, Phys. Scr., 36, 602

Humphreys, C. J. 1939, J. Res. Natl. Bur. Stand. (US), 22, 19

Humphreys, C. J., de Bruin, T. L., \& Meggers, W. F. 1931, J. Res. Natl. Bur. Stand. (US), 6, 287

Kramida, A., \& Fuhr, J. R. 2015, http://physics.nist.gov/cgi-bin/ ASBib1/TransProbBib.cgi, last updated March 2015, National Institute of Standards and Technology, Physical Measurement Laboratory, Quantum Measurement Division

Peláez, R. J., Djurovic, S., Cirisan, N., et al. 2009a, J. Phys. B: At. Mol. Opt. Phys., 42, 12, 7

Peláez, R. J., Cirisan, N., Djurovic, S., et al. 2009b, A\&A, 507, 1697

Persson, W., Wahlström, C.-G., Bertuccelli, G., et al. 1988, Phys. Scr., 38, 347

Sahal-Bréchot, S., Dimitrijevic, M. S., \& Ben Nessib, N. 2014, Atoms 2, 225

Saloman, E. B. 2004, J. Phys. Chem. Ref. Data, 33, 765

Sobelman, I.I., Vainshtein, L. A., \& Yukov, E. A. 1995, Excitation of atoms and broadening of spectral lines (Springer)

Yüce, K., Castelli, F., \& Hubrig, S. 2011, A\&A, 528, A37 